\title{
Relaciones entre los estilos de crianza actuales, el empleo de las técnicas de orientación del comportamiento y su enseñanza en Odontopediatría. Revisión bibliográfica.
}

\author{
Cristina Segarra Ortells, ${ }^{1}$ (1) Marta Ribelles Llop, ${ }^{1}$ Carla Borrell García, ${ }^{1}$ (D) \\ Laura Marqués Martínez, ${ }^{1}$ [D Ana María Leyda Menéndez. ${ }^{1}$ [D
}

\begin{abstract}
Resumen: Introducción: Los cambios en los estilos de crianza de los últimos 25 años han llevado a desestimar en Odontopediatria algunas técnicas de orientación conductual (TODC) clásicas porque los padres las consideran poco apropiadas para sus hijos. Esto ha generado cambios en los programas de formación en Odontopediatría. Objetivo: Revisar las TODC más empleadas actualmente por odontopediatras, las más enseñadas en los programas de Odontología y la influencia de la aceptación parental en ello. Material y método: se realizó una revisión descriptiva de la literatura. Para establecer la base documental, constituida por 42 artículos, se realizó una búsqueda en las bases de datos: Cochrane, SciElo, Medline y Google Academy. Resultados: La familia ha experimentado un proceso de transformación con nuevos modelos de relaciones familiares y de crianza. Aunque los odontopediatras reconocen que la crianza autoritativa es la que favorece un comportamiento más positivo en el consultorio, perciben una tendencia parental hacia la permisividad. Eso ha favorecido que el empleo de algunas TODC clásicas, como control de voz o la estabilización física activa haya disminuido y la formación y entrenamiento al respecto también. Conclusiones: La formación de los odontopediatras está influenciada por el entorno socio-cultural y por aspectos ético-legales cambiantes. Ante la tendencia actual a un patrón de crianza más permisivo las TODC más enseñadas en los programas de Odontopediatría y, las más empleadas por los odontopediatras son las de comunicación. Conocer el patrón de crianza parental puede orientar al profesional para sugerir las TODC más adecuadas a cada entorno familiar.
\end{abstract}

Palabras clave: conducta infantil, odontopediatría, aprendizaje, crianza del niño.

\section{Relações entre os estilos de criação atuais, a utilização das técnicas de orientação comportamental e seu ensino em Odontopediatria. Revisão bibliográfica.}

Resumo: Introdução: As mudanças nos estilos de criação dos últimos 25 anos levaram a rejeitar algumas técnicas de orientação comportamental (TOC) clássicas em Odontopediatria porque os pais as consideravam inapropriadas para seus filhos. Isto gerou mudanças nos programas de formação em Odontopediatria. Objetivo: Revisar as TOC mais utilizadas atualmente pelos odontopediatras, as mais ensinadas nos programas de Odontologia e a influência da aceitação parental em todo isso. Material e método: Foi feita uma revisão descritiva da literatura. Para estabelecer a base documental, composta por 42 artigos, realizou-se uma pesquisa nas bases de datos: Cochrane, SciElo, Medline y Google Academy. Resultados: A família passou por um processo de transformação com novos modelos de relacionamento familiar e parental. Embora os odontopediatras reconheçam que a criação autoritativa é a que favorece um comportamento mais positivo no consultório, percebem também uma tendência dos pais à permissividade. Isso tem favorecido que o emprego de algumas TOC clássicas, como controle de voz ou a estabilização física ativa, tenha diminuído e a formação e treinamento a esse respeito também. Conclusões: A formação dos odontopediatras é influenciada pelo entorno sócio-cultural e por aspetos ético-legais mutáveis. Dada a tendência atual para um modelo de criação mais permissivo as TOC mais ensinadas nos programas de Odontopediatria e, as mais empregadas pelos odontopediatras são as de comunicação. Conhecer o modelo parental pode orientar o profissional para sugerir as TOC mais adequadas a cada ambiente familiar.

Palabra-chave: comportamento infantil, odontopediatria, aprendizagem, educação infantil.

\footnotetext{
Universidad CEU Cardenal Herrera
} 


\section{Relationships between current parenting styles, the use of behavioral guidance techniques and their teaching in Pediatric Dentistry. A bibliographic review.}

Abstract: Introduction: The changes in parenting styles in the last 25 years have led to the dismissal in pediatric dentistry of some classic behavioral guidance techniques (BGT) because parents consider them inappropriate for their children. This has generated changes in the training programs in Pediatric Dentistry. Objective: To review the BTG most currently used by pediatric dentists, the most taught in Dentistry programs and the influence of parental acceptance on it. Material and method: A descriptive review of the literature was carried out. To establish the documentary base, consisting of 42 articles, a search was carried out in the databases: Cochrane, SciElo, Medline and Google Academy. Results: The family has undergone a process of transformation with new models of family and parenting relationships. Although pediatric dentists recognize that authoritative parenting is the one that favors a more positive behavior in the dental office, they perceive a parental tendency towards permissiveness that has favored the decrease in the use of some classic BGT, such as voice control or active physical stabilization, and the learning and training about them as well. Conclusions: The training of pediatric dentists is influenced by the socio-cultural environment and by the changing ethical-legal aspects. Given the current trend towards a more permissive parenting pattern, the most taught and most used BGT in Pediatric Dentistry programs are communication techniques. Knowing the parental upbringing pattern can guide the professional to suggest the most appropriate BGT for each family environment.

Key words: child behavior, pediatric dentistry, learning, child rearing.

\section{Introducción}

Los estilos de crianza van evolucionando según se producen cambios en las sociedades y los cambios sociales acontecidos en las últimas décadas en las culturas occidentales han generado la aparición de las llamadas "familias negociadoras" en las que las relaciones entre padres e hijos son menos jerárquicas y las normas de convivencia menos rígidas, generándose niños más demandantes y exigentes, con poco respeto a la autoridad y menor aceptación de las normas establecidas ${ }^{1}$. Además, los padres tienden a participar de forma más activa en todos los aspectos de la vida de sus hijos, también y especialmente en su educación y en el cuidado de su salud, observándose por un lado una creciente falta de voluntad para permitir que otro adulto oriente la conducta de los niños y por otro, desconfianza de los profesionales que trabajan cotidianamente con sus hijos: educadores y sanitarios. ${ }^{1-4}$

En Odontopediatría, uno de los aspectos clínicos más importantes es la orientación del comportamiento (ODC) del paciente infantil, sobre todo del poco colaborador. Para llegar a realizar con éxito un tratamiento dental es necesario establecer una relación de confianza y también de respeto con el paciente y con sus padres, lo que requiere por parte del profesional conocer al niño dentro de su entorno familiar y conocer cuáles son los criterios y las circunstancias de crianza en las que está creciendo y siendo educado. ${ }^{5}$ 
Sabemos que no es posible usar las mismas estrategias de ODC con todos los pacientes pediátricos, los criterios de selección en este caso son: el momento evolutivo del niño, sus experiencias previas y su carácter. A estos criterios hemos añadido en los últimos 20 años las preferencias de los padres. Esto hace que se haya desestimado algunas técnicas, no por su falta de efectividad, sino, porque los responsables de los menores las consideran poco apropiadas para sus hijos. Este cambio en la práctica clínica ha introducido modificaciones también en las técnicas de orientación del comportamiento (TODC) que se enseñan en las facultades de Odontología tanto a nivel de grado como de postgrado, por ello la presente revisión de la literatura tuvo como objetivo revisar las TODC más empleadas actualmente por los odontopediatras, las más enseñadas en los programas formativos de Odontología y la influencia de la aceptación paterna en todo ello.

\section{Materiales y métodos}

Para dar cumplimiento al objetivo de este trabajo se llevó a cabo una revisión descriptiva de la literatura. Para ello se realizó una búsqueda bibliográfica electrónica [marzo de 2019] empleando las bases de datos científicas Cochrane, SciElo, Medline y Google Academy. Se introdujeron los siguientes términos de búsqueda junto con el operador booleano "AND": "Behavior management AND parental attitudes AND pediatric dentistry", "contemporary parents AND behavior management", "behavior guide techniques AND parenting styles", "traditional pediatric dentistry AND current pediatric dentistry AND behavior management techniques", "parenting styles AND current pediatric dentistry".

La búsqueda se acotó en el tiempo entre los años 1984 y 2018. Los criterios de inclusión establecidos fueron:

1) Artículos en inglés sobre la aceptación parental de las TODC utilizadas en la atención odontológica a pacientes pediátricos.

2) Artículos en inglés sobre el empleo de las TODC en niños por odontopediatras.

3) Artículos en inglés sobre la enseñanza de las TODC en la formación odontológica universitaria.

Se excluyeron las cartas al editor y los resúmenes de artículos.

Laintroducción de los términos de búsqueda en las bases de datos electrónicas, arrojó un total de 640 resultados (Tabla 1). Tras eliminar los duplicados, el número total se redujo a 554.

Tres investigadoras independientemente seleccionaron los 328 artículos que cumplían los criterios de inclusión mediante la lectura del título y el resumen de los mismos. De ellos, 96 fueron descartados por estar dentro de algún criterio de exclusión. Los 232 artículos restantes fueron leídos a texto completo excluyéndose 190 por no contener información relevante para esta revisión bibliográfica. Las diferencias surgidas en esta selección entre las investigadoras fueron subsanadas por consenso. La base documental quedó constituida finalmente por 42 artículos. 
Tabla 1: Resultados iniciales arrojados por las base datos al introducir los términos de búsqueda.

\begin{tabular}{lccccc}
\hline Términos de búsqueda & Medline & Cochrane & Scielo & Google Academic & Total \\
\hline $\begin{array}{l}\text { "Behavior management" } \\
\begin{array}{l}\text { AND "parental attitudes" } \\
\text { "Contemporary parents" }\end{array}\end{array}$ 0 & 0 & 0 & 129 & 129 \\
$\begin{array}{l}\text { AND "behavior management" } \\
\text { "Behavior guide techniques }\end{array}$ & 38 & 0 & 0 & 222 & 260 \\
$\begin{array}{l}\text { AND parenting styles" } \\
\text { "Traditional pediatric dentistry" }\end{array}$ & 5 & 0 & 0 & 0 & 5 \\
$\begin{array}{l}\text { AND "current pediatric dentistry" AND } \\
\text { "behaviour management techniques" }\end{array}$ & 0 & 0 & 0 & 0 & 0 \\
$\begin{array}{l}\text { "Parenting styles" } \\
\text { AND "current pediatric dentistry" }\end{array}$ & 0 & 0 & 0 & 1 & 1 \\
"Parenting styles" & & & & & \\
AND "pediatric dentistry" & 0 & 0 & 0 & 245 & 245 \\
\begin{tabular}{l} 
Total \\
\hline
\end{tabular} & 43 & 0 & 0 & 597 & 640 \\
\hline
\end{tabular}

\section{Desarrollo y discusión}

\section{Estilos de crianza familiares}

Los padres, como cuidadores primarios, ejercen una gran influencia sobre la salud emocional presente y futura de sus hijos, su personalidad, su carácter, su desarrollo cognitivo y social y su formación académica. ${ }^{6-8}$

Actualmente la familia se encuentra inmersa en un profundo proceso de transformación, relacionado, por una parte, con los nuevos roles sociales y laborales de los padres $y$, por otra, con un contexto de cambio social y de nuevos estilos de vida, generándose así también nuevos modelos de relaciones familiares. ${ }^{?}$

Otro de los grandes cambios estructurales que se ha producido en las últimas décadas en el ámbito familiar son las características de las relaciones entre padres e hijos. El advenimiento de la familia moderna en el siglo XIX supuso un paso al primer plano de las relaciones en el seno de la familia, de forma que el amor y el bienestar afectivo pasaron a constituir un fundamento de importancia capital en la organización de la convivencia y de las relaciones entre sus miembros. Este hecho fue acompañado con la emergencia de la idea de que los hijos son una fuente de realización personal. Lo cual no excluía, sin embargo, una educación, desde la perspectiva actual, bastante rígida y estricta. La disciplina, entendida como no cuestionamiento de las normas y de la autoridad establecida, era un objetivo educativo de primer orden, y la obediencia, un valor a inculcar. Esta forma de ver las relaciones familiares fue erosionándose poco a poco, sobre todo a partir de la década de los sesenta del siglo pasado. Los jóvenes y adolescentes que comenzaron a cuestionar los estilos educativos autoritarios, son los que con el tiempo han pasado a ser padres y han ido cambiando sus estilos educativos a favor de una flexibilización cada vez mayor de las normas y el establecimiento de unas relaciones cada vez más negociadas entre 
todos los miembros de la familia. Estos padres han sustituido la norma del "respeto", que regulaba las respuestas de los hijos, entendida sobre todo como obediencia y "temor" a la reacción de los padres, por la "amistad de los hijos", en la que se busca, sobre todo, tener una buena comunicación, fomentar las potencialidades y capacidades de los hijos y comprender sus necesidades y sus puntos de vista ${ }^{10}$. A este cambio en la concepción de la familia y de las relaciones en su interior, se unen los cambios sociales y de estilo de vida que han tenido lugar en los países desarrollados en los últimos 50 años y que tienen que ver, no solo con la incorporación de la mujer al mundo laboral sino con un contexto estresante para todos, hombres y mujeres, caracterizado por altas cargas de trabajo, horarios poco compatibles con la vida familiar y déficit de tiempos compartidos con los hijos. Actualmente la organización de los tiempos familiares se ha vuelto cada vez más compleja. ${ }^{9}$

Estos factores son los que condicionan las circunstancias en los patrones de crianza actuales: crianza inconsistente (a veces laxa o sobre-reactiva), comunicación más negativa entre las partes, disminución de la supervisión del niño por los padres, establecimiento de normas y límites sobre el comportamiento del niño poco claros, padres más reactivos y poco proactivos. ${ }^{11}$

De este modo autores como Baumrind ${ }^{8}$ han resaltado en sus investigaciones que estos cambios acontecidos en los estilos de crianza han provocado también cambios en los comportamientos infantiles, observándose una relación directa entre el modo en el que los padres tratan a sus hijos $y$ el modo en el que estos se comportan
(Tabla 2). Se ha relacionado el patrón de crianza con variables como el rendimiento académico, la autoestima, la autoconfianza, la agresividad, el comportamiento delincuente o incluso el abuso de drogas, entre otras. Por lo tanto, conociendo el modo de trato paterno, es decir, el patrón de crianza, podemos predecir con bastante exactitud el modo de comportamiento de los niños. En líneas generales podemos diferenciar los siguientes tipos de padres en función del trato a sus hijos. ${ }^{10}$

a) Los padres autoritarios. Son los que valoran el control y la obediencia incuestionable. Tratan de hacer que los niños se sujeten a una norma de conducta establecida y los castigan arbitraria y enérgicamente cuando la infringen. Son más desapegados y menos cálidos que otros padres. Los niños suelen manifestar más descontento, retraimiento y desconfianza.

b) Los padres permisivos 0 sobreindulgentes. Son aquellos que valoran la autoexpresión y autorregulación de sus hijos. Exigen poco y dejan que los niños revisen sus propias actividades. Cuando tienen que imponer reglas, explican las razones. Consultan a los hijos las decisiones relacionadas con las normas y pocas veces castigan. Son cálidos y no controladores. Sus hijos preescolares suelen ser inmaduros. De hecho, son los que menos control ejercen sobre su persona y los que menos exploran. El resultado es un niño exigente, con el cual es muy difícil congeniar.

c) Los padres autoritativos. Son los que valoran la individualidad de sus hijos, pero también señalan las limitaciones 
Tabla 2: Relación entre el estilo de crianza parental, el comportamiento esperado en el niño mayor de 3 años, las TODC propuestas clásicamente y en la actualidad para el abordaje del paciente. ${ }^{12,13,15}$

\begin{tabular}{|c|c|c|c|}
\hline $\begin{array}{l}\text { Estilo de crianza } \\
\text { parental }\end{array}$ & $\begin{array}{l}\text { Comportamiento esperado } \\
\text { en el niño mayor de } 3 \text { años }\end{array}$ & $\begin{array}{l}\text { TODC clásicas propuestas para el } \\
\text { abordaje del paciente }\end{array}$ & $\begin{array}{l}\text { TODC propuestas actualmente para el } \\
\text { abordaje del paciente }\end{array}$ \\
\hline Autoritario & $\begin{array}{l}\text { Descontento, temeroso, } \\
\text { retraído y desconfiado }\end{array}$ & $\begin{array}{l}\text { Básicas } \\
\text { DMH } \\
\text { Modelado } \\
\text { Desensibilización } \\
\text { Distracción } \\
\text { Refuerzo positivo } \\
\text { Presencia/ausencia padres } \\
\text { Avanzadas } \\
\text { Sedación con óxido nitroso } \\
\text { Sedación farmacológica por vía oral }\end{array}$ & $\begin{array}{l}\text { Básicas } \\
\text { DMH } \\
\text { Modelado } \\
\text { Desensibilización } \\
\text { Distracción } \\
\text { Refuerzo positivo } \\
\text { Presencia/ausencia padres } \\
\text { Avanzadas } \\
\text { Sedación con óxido nitroso } \\
\text { Sedación farmacológica por vía oral }\end{array}$ \\
\hline Permisivo & $\begin{array}{l}\text { Inmaduro y exigente, } \\
\text { con dificultad para } \\
\text { establecimiento de vínculos }\end{array}$ & $\begin{array}{l}\text { Básicas } \\
\text { DMH y establecimiento de límites } \\
\text { Modelado } \\
\text { Distracción } \\
\text { Refuerzo positivo y negativo } \\
\text { Presencia/ausencia padres } \\
\text { Control de voz } \\
\text { Mano sobre boca } \\
\text { Avanzadas } \\
\text { Sedación farmacológica por vía oral }\end{array}$ & $\begin{array}{l}\text { Básicas } \\
\text { DMH } \\
\text { Modelado } \\
\text { Distracción } \\
\text { Refuerzo positivo } \\
\text { Padres siempre presentes } \\
\text { Sedación con óxido nitroso } \\
\text { Avanzadas } \\
\text { Sedación farmacológica por vía oral } \\
\text { Anestesia general }\end{array}$ \\
\hline Autoritativo & $\begin{array}{l}\text { Confiado, curioso, con } \\
\text { iniciativa, seguro de si mismo }\end{array}$ & $\begin{array}{l}\text { Básicas } \\
\text { DMH } \\
\text { Modelado } \\
\text { Distracción } \\
\text { Refuerzo positivo }\end{array}$ & $\begin{array}{l}\text { Básicas } \\
\text { DMH } \\
\text { Modelado } \\
\text { Distracción } \\
\text { Refuerzo positivo }\end{array}$ \\
\hline Ansioso & Tímido, cobarde & $\begin{array}{l}\text { Básicas } \\
\text { DMH } \\
\text { Modelado } \\
\text { Desensibilización } \\
\text { Distracción } \\
\text { Refuerzo positivo } \\
\text { Control de voz } \\
\text { Presencia/ausencia padres } \\
\text { Avanzadas } \\
\text { Sedación farmacológica por vía oral }\end{array}$ & $\begin{array}{l}\text { Básicas } \\
\text { DMH } \\
\text { Modelado } \\
\text { Desensibilización } \\
\text { Distracción } \\
\text { Refuerzo positivo } \\
\text { Padres siempre presentes } \\
\text { Sedación con óxido nitroso } \\
\text { Avanzadas (consideradas en ocasiones } \\
\text { como de primera opción) } \\
\text { Sedación farmacológica por vía oral } \\
\text { Anestesia general }\end{array}$ \\
\hline Que rechazan & Desobediente, patalea & $\begin{array}{l}\text { Básicas } \\
\text { DMH } \\
\text { Modelado } \\
\text { Desensibilización } \\
\text { Distracción } \\
\text { Refuerzo positivo y negativo } \\
\text { Presencia/ausencia padres } \\
\text { Control de voz } \\
\text { Mano sobre boca } \\
\text { Avanzadas } \\
\text { Sedación farmacológica por vía oral }\end{array}$ & $\begin{array}{l}\text { Básicas } \\
\text { DMH } \\
\text { Modelado } \\
\text { Desensibilización } \\
\text { Distracción } \\
\text { Refuerzo positivo } \\
\text { Padres prefieren estar fuera del gabinete } \\
\text { Control de voz } \\
\text { Sedación con óxido nitroso } \\
\text { Avanzadas } \\
\text { Sedación farmacológica por vía oral }\end{array}$ \\
\hline Sobre-afectivo & Desobediente, exigente & $\begin{array}{l}\text { Básicas } \\
\text { DMH y establecimiento de límites } \\
\text { Modelado } \\
\text { Distracción } \\
\text { Refuerzo positivo y negativo } \\
\text { Presencia/ausencia padres } \\
\text { Control de voz } \\
\text { Mano sobre boca } \\
\text { Avanzadas } \\
\text { Sedación farmacológica por vía oral }\end{array}$ & $\begin{array}{l}\text { Básicas } \\
\text { DMH } \\
\text { Modelado } \\
\text { Desensibilización } \\
\text { Distracción } \\
\text { Refuerzo positivo } \\
\text { Padres siempre presentes } \\
\text { Sedación con óxido nitroso } \\
\text { Avanzadas (consideradas en ocasiones } \\
\text { como de primera opción) } \\
\text { Sedación farmacológica por vía oral } \\
\text { Anestesia general }\end{array}$ \\
\hline
\end{tabular}


sociales. Confían en su capacidad para orientarlos, pero también respetan las decisiones de independencia, los intereses, las opiniones y la personalidad de los niños. Son cariñosos e indulgentes, pero también exigen un buen comportamiento; mantienen con firmeza las normas e imponen con sensatez castigos limitados cuando es necesario en contexto de una relación cálida y de apoyo.

d) Lospadresansiosos. Son los que proceden de familias donde ha habido muertes o en el caso de ser padres muy jóvenes o inexpertos. Suelen ejercer sobre el niño sobre-afecto y sobreprotección motivados por el temor y la ansiedad. El niño depende de sus padres para tomar decisiones y emprender actividades y normalmente responde con timidez y cobardía ante situaciones nuevas.

e) Los padres que rechazan. El origen de esta situación suele estar en causas como celos, mala situación económica, inmadurez, etc. Actúan generalmente alejados del niño, manteniendo con él una actitud negativa de crítica, de castigo, de disciplina exagerada e inconstante. El resultado suele ser un niño desobediente, que puede pretender una capacidad de mando inexistente, propenso a pataletas, a ser mentiroso, o incluso a robar.

f) Los padres sobre-afectivos. Son aquellos que han tenido a sus hijos en edad avanzada o es hijo único, hijo adoptado o el menor de la familia. Son niños con una preparación inadecuada para ocupar su debido lugar en la sociedad, en la escuela o en el hogar.
La evidencia científica indica que la tolerancia al estrés dental y las habilidades de afrontamiento de los niños son mejores cuandohayunentorno familiarestructurado y de cordialidad y aceptación, los padres son sensibles y seguros de si mismos, aportan un buen soporte emocional a sus hijos con un grado de control intermedio, estableciendo límites claros y ofreciendo recompensas y castigos apropiados, es decir, son padres autoritativos. Los hijos son curiosos, confían en si mismos, tienen iniciativa y suelen mostrar un buen funcionamiento escolar. ${ }^{10}$

2. Percepción de los odontopediatras sobre los estilos de crianza de sus pacientes pediátricos

Como se ha mencionado en la introducción, actualmente los odontopediatras son testigos de la evolución de los cambios sociales acontecidos en los últimos 25 años, especialmente con respecto a los estilos de crianza. Algunos estudios ${ }^{1-3}$, muestran que los profesionales tienen la percepción de que los padres contemporáneos están más ocupados laboralmente que en generaciones anteriores y de que sus hijos presentan más dificultad para aceptar normas o para identificar autoridad en una persona adulta, incluidos sus propios padres. Casamassimo et al. ${ }^{1}$ recogieron la percepción de los odontólogos en relación a los cambios en los estilos de crianza observando que los profesionales los describían como más permisivos y menos autoritarios, junto con una creciente falta de voluntad de los padres para permitir que otro adulto oriente la disciplina o el comportamiento de sus hijos y una desconfianza creciente de la atención médica tradicional. Los padres están más 
interesados que en décadas anteriores en el cuidado de sus hijos poniendo un gran énfasis en que tengan una experiencia odontológica positiva ${ }^{1-3}$. Son en general familias con un patrón de crianza permisivo en las que se ha observado una mayor dificultad de los niños para establecer vínculo con su odontopediatra y para colaborar con él en la realización de los tratamientos dentales.

Junto con el patrón permisivo, los profesionales también reciben pacientes de familias con estilos de crianza autoritarios y autoritativos. Las primeras, cada vez menos numerosas, generan unos niños más temerosos, menos espontáneos y esto en muchos casos desemboca en comportamientos poco colaboradores en el consultorio ${ }^{13}$. Sin embargo, los odontopediatras perciben que la crianza autoritativa proporciona un impacto más positivo en los niños, (en comparación con los estilos de crianza autoritario y permisivo), favoreciendo un mejor desarrollo emocional y un comportamiento más positivo en el consultorio dental. 8,12,13 $^{2}$

3. Evolución de la formación universitaria en TODC básicas aplicadas en Odontopediatría y su aceptación parental

LasTODC son unmétodo integralycontinuo destinado a desarrollar y fortalecer la relación entre el odontopediatra y el niño, favoreciendo la confianza y la comunicación y disminuyendo el miedo y la ansiedad. La Academia Americana de Odontopediatría (AAPD) clasifica las principales TODC en básicas y avanzadas ${ }^{14}$ (Tabla 3 ).

La elección de las TODC a utilizar dependerá de los conocimientos, la formación, la experiencia, la filosofía, la capacidad de
Tabla 3: Clasificación de las TODC según la $A A P D^{14}$.

\begin{tabular}{ll}
\hline Técnicas básicas & Técnicas avanzadas \\
\hline $\begin{array}{l}\text { Decir / Mostrar / Hacer } \\
\text { (DMH) }\end{array}$ & Estabilización protectora \\
Modelado & Sedación farmacológica \\
Imágenes positivas antes & Anestesia general \\
de la visita & \\
Control de voz & \\
Comunicación no verbal & \\
Refuerzo positivo y elogio \\
descriptivo \\
Distracción \\
Ausencia o presencia de \\
los padres \\
$\begin{array}{l}\text { Sedación con óxido } \\
\text { nitroso }\end{array}$
\end{tabular}

cada odontopediatra, las características del paciente, así como de las preferencias de los padres. ${ }^{15}$

Hoy en día, ha disminuido no solamente el empleo de algunas TODC que tradicionalmente eran utilizadas en la clínica cuando algunos niños lo requerían sino también, la formación al respecto en los programas de Odontopediatría universitarios, ya que estas técnicas son consideradas actualmente como inadecuadas, peligrosas o maltratantes ${ }^{16}$ (Tabla 2). En los programas de postgrado en Odontopediatría ahora se enseñan menos las técnicas consideradas aversivas en comparación con el uso de aquellas comunicativas o farmacológicas, lo que hace que los odontólogos más jóvenes empleen con más frecuencia técnicas como: Decir / Mostrar / Hacer (DMH) o el refuerzo positivo a través de recompensas y elogios ${ }^{15-19}$. Adair et al. ${ }^{18}$ en el año 2004 observaron que en el $98 \%$ de los postgrados de Odontopediatría en Estados Unidos (EE.UU.) se enseñaban las técnicas 
de comunicación como aceptables, con la excepción de la técnica mano sobre boca, considerada como inaceptable en el 54\% de los programas. Todos estos programas formaban, además, para el uso de las técnicas farmacológicas (óxido nitroso, sedación, anestesia general) considerándolas también como aceptables. Estos resultados son coherentes con el hecho de que DMH es la técnica de comunicación más utilizada universalmente por odontopediatras y la que presenta mayor aceptación por parte de los padres $4,5,16,19-21$. Prácticamente el $100 \%$ de los odontólogos especializados en atención pediátrica la usan cotidianamente con todos sus pacientes ${ }^{22,23}$.

Sin embargo, la técnica denominada control de voz, consistente en alterar conscientemente la voz a través de cambios en su volumen, ritmo y tono para influir directamente en el comportamiento del paciente, con el fin de ganar su atención y confianza, muestra una clara tendencia a la disminución en su uso por odontopediatras más jóvenes, recién graduados ${ }^{20,24}$. Wells et al. ${ }^{25}$ (2018) afirmaron que los profesionales con más años de experiencia recibieron formación en un momento en que el uso del control de voz se consideraba menos discutible, siendo más propensos a utilizarlo en su práctica diaria. El aumento del tono de la voz puede ser considerado aversivo o maltratante por parte de los padres, ya que hoy en día algunos nunca han levantado la voz a sus hijos. Eaton ${ }^{15}$ (2005) y Patel ${ }^{16}$ (2016) observaron, en sus respectivos estudios, que esta técnica era considerada como agresiva y los padres preferían el empleo de sedación o anestesia general. Aunque todavía está incluida en las guías de la AAPD y aún se utiliza en pacientes pediátricos, se recomienda explicar a los padres antes de realizar el tratamiento en qué consiste para evitar posibles malos entendidos. ${ }^{14}$

La presencia o ausencia de los padres en el gabinete dental, es una técnica consistente en requerir que los padres salgan temporalmente ante un mal comportamiento del paciente y que puedan volver a entrar cuando el niño comience a colaborar positivamente con el odontólogo. Tradicionalmente, se ha mantenido a los padres fuera del consultorio durante el tratamiento dental de sus hijos, eliminando muchos problemas de comportamiento en los pacientes, ya que permite al odontopediatra establecer una relación más cercana con ellos y disminuir la ansiedad, particularmente si los padres presentan miedo dental ${ }^{20}$. Sin embargo, los padres contemporáneos prefieren estar presentes para observar todos los procedimientos que se realicen; esta tendencia se ha observado en distintos países y culturas ${ }^{26,27}$. Esto ha hecho que en los últimos años se observe una mayor tendencia de los odontopediatras a permitir la presencia parental durante la visita ${ }^{11,24}$. Sin embargo, sigue siendo esta una cuestión de preferencia personal que durante mucho tiempo ha estado en controversia entre los propios profesionales. En algunos casos y contextos la separación de los padres durante el tratamiento odontológico es una de las técnicas con menor aceptación por parte de los progenitores ${ }^{20}$, mientras que Boka et al. ${ }^{21}$ (2014) en su estudio realizado en Grecia indicaron que fue la segunda TODC más aceptada por los padres (78\%).

La ausencia puntual de los padres puede ser una TODC eficaz y tiene alta aceptación por los progenitores si se dialoga con ellos antes de realizar el tratamiento ${ }^{28,29}$. Así se enseña actualmente en las facultades de 
Odontología como informaron York et al. ${ }^{22}$ (2007), quienes observaron que el $60 \%$ de los estudiantes de grado había recibido formación teórica sobre esta técnica, el $34,7 \%$ la había observado realizar y el 33,7\% la utilizaba en la práctica clínica.

En los últimos años se ha observado un incremento en el uso de la sedación inhalatoria con óxido nitroso. Este aumento es debido a la buena aceptación de su empleo por parte de los padres por considerarla una técnica poco aversiva que respeta al niño y le evita sufrimiento y estrés. Estudios recientes muestran que tras DMH, esta es la técnica más aceptada por los padres que esperan que la sedación con óxido nitroso facilite una experiencia dental positiva. ${ }^{16,20,28,30}$

Es posible que el aumento del uso del óxido nitroso sea también el reflejo de la educación que se imparte actualmente a los odontopediatras. Los profesionales jóvenes se sienten bien formados y bien preparados para utilizar esta técnica y totalmente dispuestos a hacerlo ${ }^{24,31}$. Ya en el año 2007 en EE.UU. el 93\% de los estudiantes de Odontología fueron instruidos para intervenir farmacológicamente utilizando óxido nitroso, benzodiacepinas u otros fármacos sedantes para la orientación del comportamiento de los pacientes pediátricos ${ }^{23}$.

4. Evolución de la formación universitaria en TODC avanzadas aplicadas en Odontopediatría y su aceptación paterna.

La aceptación por parte de los padres de las TODC avanzadas está en evolución, como se observa en la Figura 1. El uso de técnicas farmacológicas tiene cada vez mayor aprobación y menor la estabilización protectora. Los padres más permisivos y sobreprotectores aceptan, e incluso solicitan TODC farmacológicas tales como sedación o anestesia general por percibirlas como menos arriesgadas y más cómodas para sus hijos, evitándoles una situación que ellos mismos pueden considerar desagradable e incluso potencialmente traumática ${ }^{32-35}$. Sin embargo, los padres autoritativos tienden a rechazar estas técnicas o a tenerlas en cuenta con mucha reserva ${ }^{12}$.

Los estudios realizados muestran que la sedación farmacológica por vía oral en la mayoría de los contextos en los últimos 36 años no es considerada por los padres como una técnica de primera opción, por lo que está situada en un orden de aceptación del

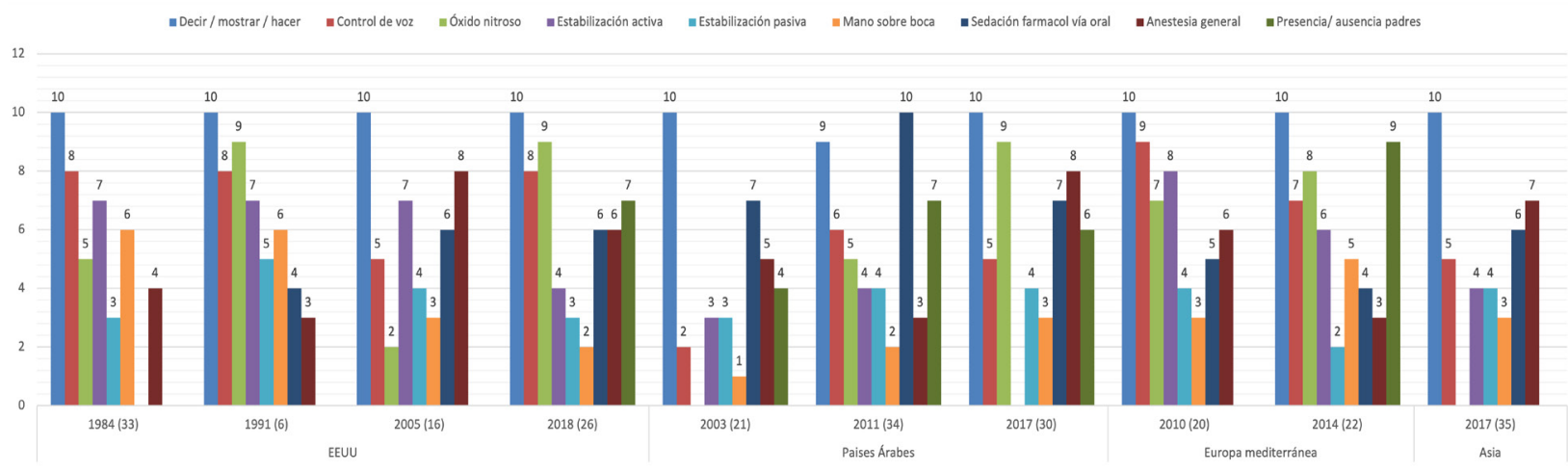

Figura 1. Evolución del grado de aceptación del uso de las técnicas de orientación de la conducta en diferentes contextos geográficos. 
1 al 10 (1 la más aceptada, 10 la menos) siempre a partir del $5^{\circ}$ lugar $5,19,21,34$ (Figura 1). La única excepción en la literatura revisada la encontramos en Arabia Saudí29,33, país en el que la sedación farmacológica por vía oral ocupó el $4^{\circ}$ puesto en el orden de aceptación de las TODC.

La estabilización protectora, si no es bien utilizada y no hay una buena comunicación con los padres y un consentimiento previo, puede ser interpretada por ellos como una agresión contra el paciente pediátrico, por lo que requiere una exhaustiva explicación tanto del porqué es necesaria como de la manera de instaurarla ${ }^{22}$. Además, es imprescindible la aceptación explícita de los padres de su uso y la firma del consentimiento informado ${ }^{27,36}$.

En general, es posible decir que los padres se muestran mucho más disponibles a aceptar las TODC básicas o avanzadas cuando éstas son explicadas previamente a su aplicación en el niño $0^{5,16,21,37}$, incluso pudiendo llegar a reconocer la necesidad de su uso y solicitándolas al profesional ${ }^{22}$.

No se ha encontrado en la bibliografía revisada asociaciones entre la aceptación de las TODC y el género o la edad de cualquiera de los progenitores ${ }^{5,16,22,25,32}$ o del paciente pediátrico ${ }^{32}$, ni correlación entre la aceptación de las TODC avanzadas por parte de los padres y la experiencia dental previa de los padres o del niño ${ }^{22,32}$.

Las TODC avanzadas requieren de formación específica por parte del odontólogo para poder utilizarlas de forma segura y eficaz ${ }^{38}$. La sedación farmacológica por vía oral y la anestesia general, son las más utilizadas por odontopediatras recién graduados en comparación con profesionales formados ya hace varias década ${ }^{\mathrm{s} 20}$.

Actualmente, más del $75 \%$ del alumnado de Odontología estudia teóricamente durante el grado qué es la estabilización protectora: sus tipos, sus indicaciones y su uso, pero la ausencia de entrenamiento práctico supone una formación limitada y la falta de las competencias necesarias para su práctica clínica ${ }^{23}$. Esto es muy importante porque los intentos de utilizarla sin una formación completa pueden poner en riesgo de daño físico al paciente, al odontólogo y a su equipo, además de las repercusiones psicológicas que un mal uso de esta técnica puede generar en el niño, en el profesional y en los padres. ${ }^{36}$

Adair et al. ${ }^{17}$ (2004) observaron que el $42 \%$ de las instituciones encuestadas en EE.UU. afirmaron que el $25 \%$ de los estudiantes de postgrado tenía experiencia práctica en el uso de la estabilización protectora pasiva en pacientes no sedados, mientras que el $27 \%$ de los programas no proporcionó estas experiencias.

En relación a la sedación farmacológica por vía intravenosa su porcentaje de uso es menor que el de la vía oral ${ }^{38}$. En torno al $50 \%$ de los odontopediatras reciben formación específica teórica para el uso de esta técnica, pero el porcentaje de profesionales entrenados en la práctica es menor ${ }^{15}$ y como resultado menos de un cuarto de ellos la usa habitualmente para tratar pacientes pediátricos ${ }^{34,39}$.

Con respecto a la anestesia general, es considerada una alternativa factible en la atención odontopediátrica ${ }^{39}$. El aumento de su uso para procedimientos 
médicos en pacientes pediátricos y su mayor aceptación por parte de los padres, puede estar influyendo en la frecuencia con que los profesionales más jóvenes ofrecen esta posibilidad para la atención de algunos pacientes, especialmente niños muy pequeños, de comportamiento no colaborador o con diversidad cognitiva. A medida que la experiencia profesional aumenta, los estudios refieren que el uso de la anestesia general disminuye $\mathrm{e}^{24,40-42}$.

Diversos autores han sugerido en los últimos 20 años la necesidad de cambios en los programas formativos en relación con la enseñanza de las TODC, basándose en los cambios sociales, la seguridad percibida y la evidencia científica disponible al respecto en la actualidad. ${ }^{16,18,21}$

Entre los cambios que ya son evidenciables, el más significativo es el acontecido en relación al uso de la técnica mano sobre boca. Aunque fue eliminada de las guías clínicas de la AAPD en el año 2006, una encuesta realizada en el 2010 a los miembros de esta academia registró que 320 de los 704 odontólogos encuestados (50\%), creía que la técnica seguía siendo aceptable ${ }^{33}$. Actualmente la mayoría de los programas no la enseñan y su uso ha perdido mucha aceptación en la práctica clínica ${ }^{17,23,42}$.

\section{Conflicto de intereses}

Todas las autoras declaran no tener ningún conflicto de interés en la realización de este estudio.

\section{Conclusiones}

La formación de los odontopediatras está influenciada por los constantes cambios en el entorno socio-cultural y en el ámbito éticolegal acontecidos en las dos últimas décadas.

Ante la tendencia actual a un patrón de crianza más permisivo el comportamiento exhibido por los pacientes pediátricos tiende a ser inicialmente menos colaborador.

Algunas de las técnicas que clásicamente se hubiesen utilizado para orientar el comportamiento de estos niños son menos usadas por ser consideradas por los padres inadecuadas prefiriendo incluso el uso de técnicas avanzadas.

Las técnicas más enseñadas en los programas de Odontopediatría, las más empleadas por los odontopediatras y las más aceptadas por los padres son las técnicas básicas encaminadas al establecimiento de la comunicación.

Conocer el patrón de crianza parental puede orientar al profesional para sugerir las TODC más adecuadas a cada entorno familiar.

\section{Referencias bibliográficas}

1. Casamassimo P, Wilson S, Gross L. Effects of changing US parenting styles on dental practice: perceptions of diplomates of the American Board of Pediatric Dentistry. Pediatr Dent 2002;24:18-22.

2. Harper DC, Donna P. The child's voice: understanding the contexts of children and families today. Pediatr Dent 2004;26:114-20. 
3. Sheller B. Challenges of managing child behavior in the 21st century dental setting. Pediatr Dent 2004;26:111-3.

4. Roberts JF, Curzon MEJ, Koch G, Martens LC. Review: Behavior management techniques in pediatric dentistry. Eur Arch Paediatric Dent 2010;11:166-74.

5. Lawrence SM, McTigue DJ, Wilson S, Odom JG, Waggoner WF, Fields HW Jr. Parental attitudes toward behavior management techniques used in pediatric dentistry. Pediatr Dent 1991;13:151-5.

6. DeVore ER, Ginsburg KR. The protective effects of good parenting on adolescents. Curr Opin Pediatr 2005;17:460-5.

7. Law CS. The impact of changing parenting styles on the advancement of peadiatric oral health. J Calif Dent Assoc 2007;35:192-7.

8. Baumrind D. Current Patterns of parental authority. Dev Psychol Monogr 1971;4:1-103.

9. Long N. The changing nature of parenting in America. Pediatr Dent 2004;26:111-3.

10. Melamed BG, Ross SL, Courts F, Bennett CG, Jerrell G, Ross L, et al. Dentists behavior management as it affects compliance and fear in pediatric patients. J Am Dent Assoc 1983; 106(3): 324-30.

11. Deater-Deckard K. Parenting stress and child adjustment: Some old hypotheses and new questions. Clin Psychol 1998;5:314-32.

12. Taran PK, Kaya MS, Bakkal M, Özalp S. The effect of parenting styles on behaviour management technique preferences in a Tuskish population. Pediatr Dent 2018;15:40360-4.

13. Aminabadi NA, Farahani RM. Correlation of parenting style and pediatric behavior guidance strategies in the dental setting: preliminary finding. Act Odontol Scand 2008;66:99-104.

14. American Academy of Pediatric Dentistry. Guideline on behavior guidance for the pediatric dental patient. Pediatr Dent 2018;32:147-55.

15. Eaton JJ, McTigue DJ, Fields HW Jr, Beck M. Attitudes of contemporary parents toward behavior management techniques used in pediatric dentistry. Pediatr Dent 2005;27:107-13.

16. Patel, McTigue DJ, Thikkurissy S, Fields HW. Parental attitudes toward advanced behavior guidance techniques used in pediatric dentistry. Pediatr Dent 2016;38:30-6.

17. Adair SM, Waller JL, Schafer TE, Rockman RA. A survey of members of the American Academy of Pediatric Dentistry on their use of behavior management techniques. Pediatr Dent 2004;26:159-66.

18. Wright FA, McMurray NE, Giebartowski J. Strategies used by dentist in Victoria, Australia, to manage children with anxiety or behavior problems. J Dent Child 1991;58:223-8.

19. De León JL, Guinot-Jimeno F, Bellet-Dalmau LJ. Acceptance by Spanish parents of behavior management techniques used in pediatric dentistry. Eur Arch Pediatr Dent 2010;11:175-8.

20. Abushal M, Adenubi JO. Attitudes of Saudi parents toward behavior management techniques in pediatric dentistry. J Dent Child 2003;70:104-10.

21. Boka V, Arapostathis K, Vretos N, Kotsanos N. Parental acceptance of behavior-management techniques used in pediatric dentistry and its relation to parental dental anxiety and experience. Eur Arch Pediatr Dent 2014;15:333-9.

22. York K, Mlinac M, Deibler M, Creed T, Ganem I. Pediatric behavior management techniques: A survey of predoctoral dental students. J Dent Edu 2007;71:532-9.

23. Adair SM, SchaferTE, Waller JL, Rockman RA. Age and gender differences in the use of behavior management techniques by pediatric dentist. Pediatr Dent 2007;29:403-8.

24. Shroff S, Hughes C, Mobley C. Attitudes and preferences of parents about being present in the dental operatory. Pediatr Dent 2015;37:51-5.

25. Wells MH, McCarthy BA, Tseng CH, Law CS. Usage of behavior guidance techniques differs by provider and practice characteristics. Pediatr Dent 2018;40:201-7.

26. Arathi R, Ashwini R. Parental presence in the dental operatory: parents point of view. J Indian Soc Pedod Rev Dent 1999;17:150-5.

27. Oliver K, Manton DJ. Contemporary behavior management techniques in clinical pediatric dentistry: out with the old and in with the new? J Dent Child (Chic) 2015;82:22-8.

28. Kotsanos N, Arhakis A, Coolidge T. Parental presence versus absence in the dental operatory: a technique to manage the uncooperative child dental patient. Eur J Pediatr Dent 2005;6:144-8.

29. Al Daghamin S, Balharith M, Alhazmi S, Al Obaidi F, Kakti A. Behavior management techniques in pediatric dentistry: How well are they accepted?. Acad J Ped Neonatol 2017;5:1-6.

30. Wilson S, Houpt M. Project USAP 2010: Use of sedative agents in pediatric dentistry a 25 year follow up survey. Pediatr Dent 2016;38:127-33. 
31. Wilson S, Cody WE. An analysis of behavior management papers published in dental literature. Pediatr Dent 2004;27:331-8.

32. Murphy MG, Fields HW, Manchen JB. Parental acceptance of pediatric dentistry behavior management techniques. Pediatr Dent 1984;6:193-8.

33. Muhammad S, Shyama M, Al-Mutawa SA. Parental attitude toward behavioral management techniques in dental practice with school children in Kuwait. Med Princ Pract 2011;20:350-5.

34. Acharya S. Parental acceptance of various behavior management techniques used in pediatric dentistry: $A$ pilot study in Odisha, India. Pesq Bras Odontoped Clin Integr 2017;17:e3728. Disponible en: http://revista. uepb.edu.br/index.php/pboci/article/view/3728

35. American Academy of Pediatric Dentistry. Guideline on use of nitrous oxide for pediatric dental patients. Pediatr Dent 2014;36:204-8.

36. Olabi NF, Jones JE, Saxen MA, Sanders BJ, Walker LA, Weddell JA, et al. The use of office based sedation and general anesthesia by board certified pediatric dentist practicing in the United States. Anesth Prog 2012;59:12-7.

37. Nunn J, Foster M, Master S, Greening S, Bristish Society of Pediatric Dentistry: A policy document on consent and the use of physical intervention in the dental care of children. Int J Pediatr Dent 2008;18(suppl 1):39-46.

38. De Castro AM, de Oliveira FS, de Paiva Novaes MS, Araujo Ferreira DC. Behavior guidance techniques in Pediatric Dentistry: attitudes of parents of children with disabilities and without disabilities. Spec Care Dentist 2013;33:213-7.

39. Adair SM, Rockman RA, Schafer TE, Waller JL. Survey of behavior management teaching in pediatric dentistry advanced education programs. Pediatr Dent 2004;26:151-8.

40. Wilson S, Nathan JE. A survey study of sedation training in advanced pediatric dentistry programs: thoughts of program directors and students. Pediatr Dent 2011;33:231-5.

41. Keith A, Eric H, Sharon K. Comparing four methods of inform parents about child behavior management: how to inform to consent. Pediatr Dent 1995;17:180-6.

42. Lambreno K, McArthur E. Introducing a clinical holding policy. Pediatr Nurs 2003;15:30-3.

Recibido: $27 / 11 / 20$

Aceptado: 21/01/22

Correspondencia: Cristina Segarra Ortells, correo: cristina.segarra@uchceu.es 